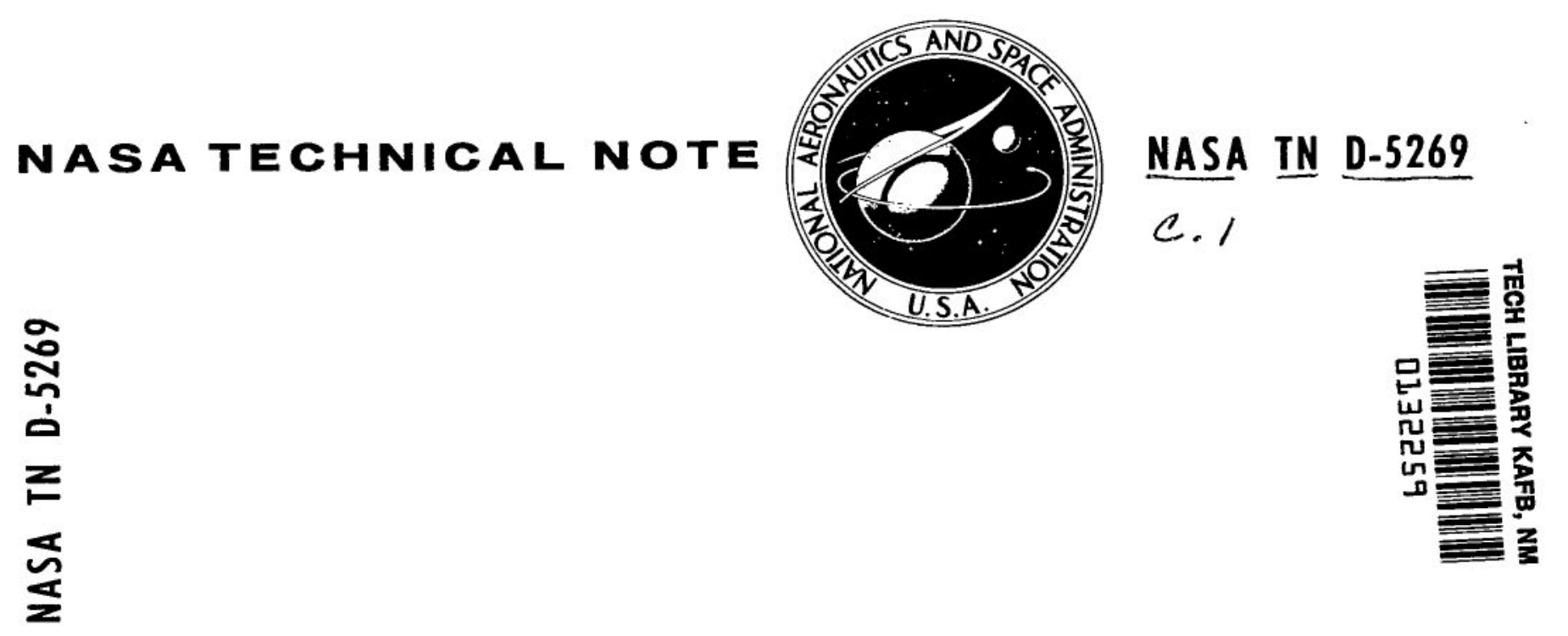

LOAN COPY: RETURN TO AFWL (WLIL-2)

KIRTLAND AFB, N MEX

\title{
PLASMA GENERATION \\ USING A LARGE V-GROOVE CATHODE DISCHARGE
}

by Paul R. Caron

Electronics Research Center

Cambridge, Mass.

NATIONAL AERONAUTICS AND SPACE ADMINISTRATION - WASHINGTON, D. C. - JUNE 1969 


\section{PLASMA GENERATION USING A \\ LARGE V-GROOVE CATHODE DISCHARGE}

By Paul R. Caron

Electronics Research Center

Cambridge, Mass.

NATIONAL AERONAUTICS AND SPACE ADMINISTRATION

For sale by the Clearinghouse for Federal Scientific and Technical Information Springfield, Virginia 22151 - CFSTI price $\$ 3.00$ 


\section{PLASMA GENERATION USING A \\ LARGE V-GROOVE CATHODE DISCHARGE}

By Paul R. Caron

Electronics Research Center

\section{SUMMARY}

The results of an investigation of the plasma parameters generated by a large V-groove cathode discharge are presented. The cathode consists of a 12-inch aluminum plate into which V-grooves of 1/8-inch spacing and 60-deg angle are milled. With this cathode, a negative glow discharge is produced which yields a stable, steady-state plasma. Electron densities up to $2 \times 10^{12}$ $\mathrm{cm}^{-3}$ have been obtained in the 12-inch diameter chamber. The electron temperature is about $1200^{\circ} \mathrm{K}$ and is independent of discharge current and gas pressure over the range 0.3 to 1.0 torr.

The discharge operates in much the same fashion as a conventional brush cathode discharge but with lower operating voltage for equal current densities. The lower operating voltage, which reduces the arcing problems at the cathode-glass junction, and the relative ease in fabricating the $\mathrm{V}$-groove cathode make it an attractive alternative to the brush cathode for many applications. It is shown that the plasma is beam-generated and the dominant electron-loss mechanism in the steady-state is electron-ion recombination. However, although most electrons are lost by recombination, diffusion losses still play a strong role in determining the electron density profile.

\section{INTRODUCTION}

For many laboratory investigations, it is desired to construct a physically large plasma of sizable electron density. In particular, these characteristics are often desirable for studying microwave propagation in plasmas where the plasma should be many free-space wavelengths thick and where the propagation characteristics for wave frequencies near the plasma frequency are desired. The V-groove cathode discharge discribed in this technical note is well suited to such investigations. It will be shown that the plasma thickness and the electron density can be varied over a wide range. The plasma temperature is constant and the electron-neutral collision frequency can be varied by about a factor of 3; however, this cannot be done independently of the other quantities. Also, the electron-ion collision frequency is at least as large as the electron-neutral collision frequency. 
The discharge operates basically as a cold-cathode, abnormal, negative glow discharge in helium. The discharge configuration is shown in Figure 1. The tube diameter and, hence, the diameter of the cathode, is approximately 12 inches. The anode-to-cathode distance can be varied at will but, for reasons which will be discussed later, the results of this report will be confined to the case wherein this distance is 6 inches. The cathode consists of an aluminum plate with milled V-grooves of 60-deg angle and 1/8-inch spacing. With these grooves, the cathode current caused by secondary emission processes (i.e., ion-bombardment and photoelectric emission) remains uniform over the cathode surface in the abnormal glow region. That is, the tendency for a localized discharge or arc to form, which appears in large, flat cathodes, is reduced. Also, the grooves increase the effective cathode area and increase the electron yield by ion-bombardment due to non-normal incidence of ions on the cathode (ref. 1).

The chamber is pumped down to low pressure and backfilled with helium. To improve the gas purity, helium is allowed to flow continuously through the chamber at a slow rate and the pressure is adjusted by means of a leak valve in series with the helium supply. A dc voltage applied between the anode and cathode produces a negative glow discharge whose behavior is qualitatively similar to that of a brush cathode discharge (ref. 2). That is, the plasma is beam-generated and the electron density is sufficiently high (i.e., order of $10^{12} \mathrm{~cm}^{-3}$ ) and the electron temperature is sufficiently low (i.e., approximately $1200^{\circ} \mathrm{K}$ ) so that electron-ion recombination* is the dominant electron loss mechanism.

The voltage-current characteristic in the abnormal glow region is shown in Figure 2 for various pressures. At low currents, there is a negative resistance phenomenon which is not displayed in these curves. These characteristics, as well as the plasma parameters, are dependent upon the helium purity and the condition of the cathode surface. Since a large volume exists with a large aluminum cathode and significant cathode and anode dissipation, the gas purity and cathode surface cleanliness are dependent upon the discharge current and the helium flow rate. No attempt was made to obtain an ultraclean cathode since, due to its size, this is nearly impossible. This discharge is intended as a means for generating steady-state, well-behaved plasmas of large density and physical size. Therefore, the

*This is most likely collisional-radiative recombination (ref. 3) with, perhaps, some disassociative recombination at the higher pressures of operation. 


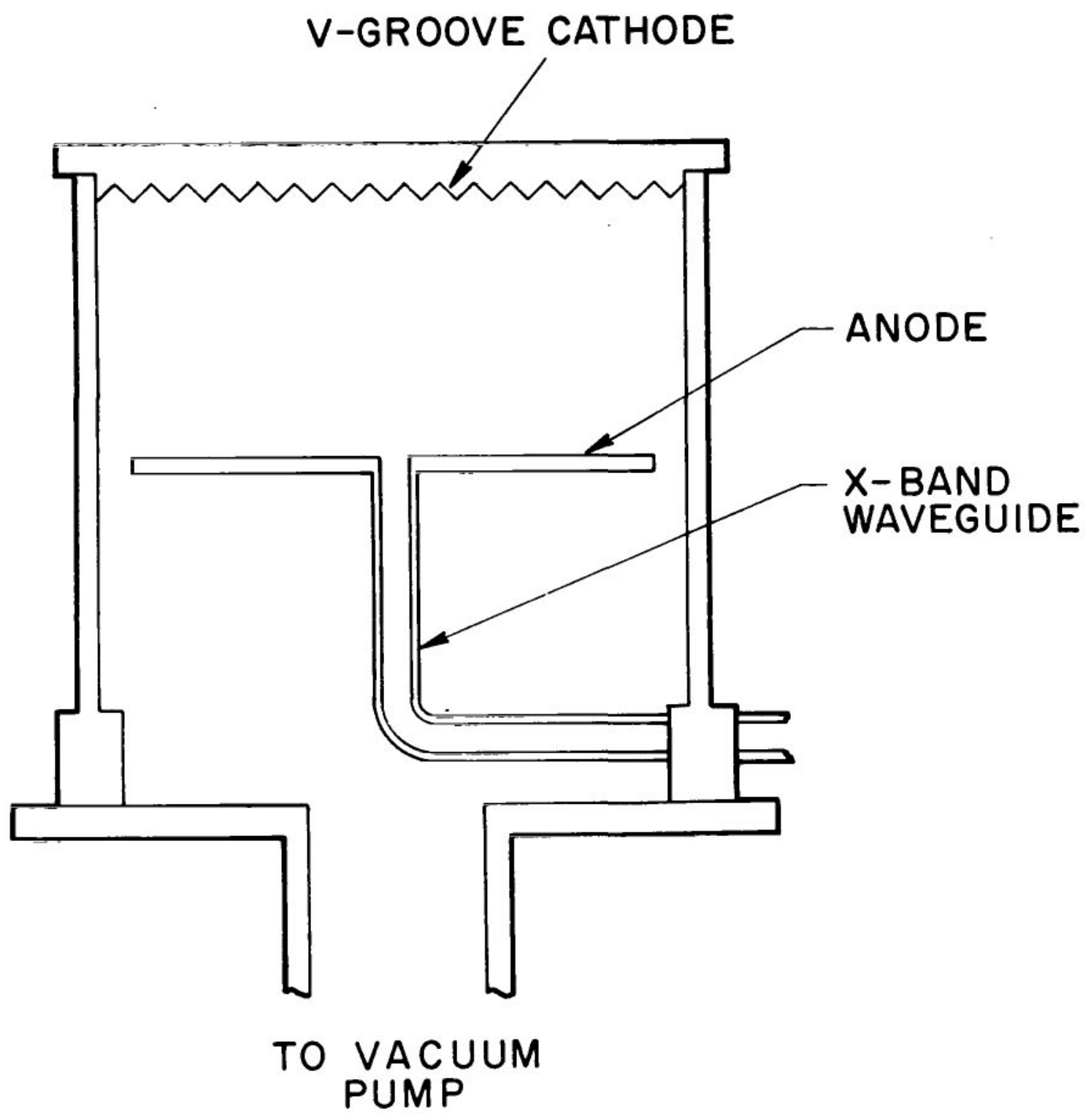

Figure 1.- Schematic of V-groove cathode discharge. 


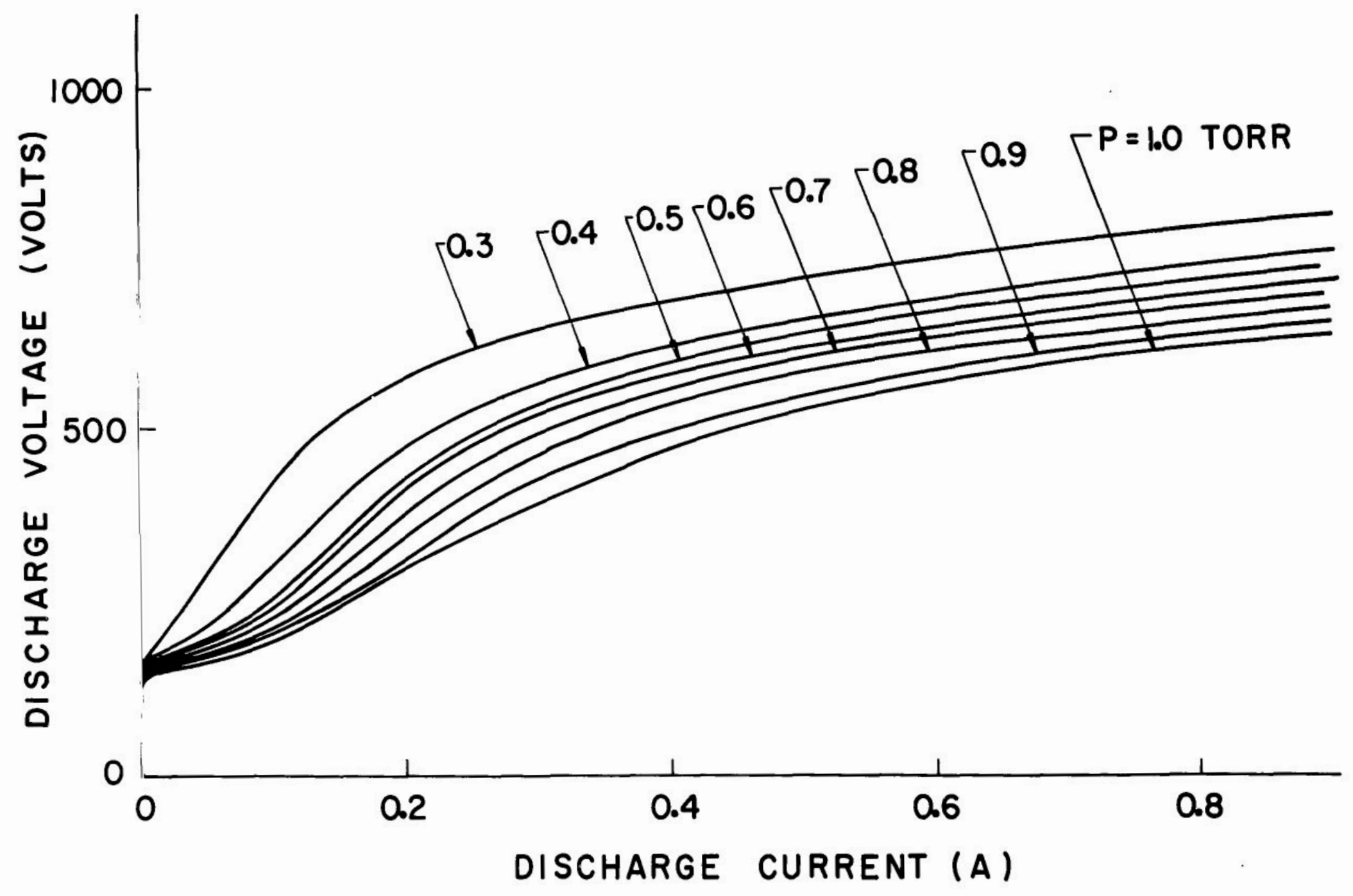

Figure 2.- Voltage-current characteristics of the V-groove cathode discharge at various pressures. 
reproducibility of the parameters is important. This reproducibility was obtained by operating the discharge with the same helium flow rate at each pressure, and only recording data at each current after the discharge had stabilized. With this procedure, excellent data reproducibility was obtained.

From Figure 2, it is noted that the operating voltage is substantially lower than that of the brush cathode discharge developed by Persson (ref. 2). This is due to the higher secondary emission coefficients of aluminium compared to these coefficients for the materials used by Persson. Thus, not only is the V-groove cathode easier to fabricate, * but it also is subject to far less cathode dissipation for the same discharge current. Furthermore, the lower beam voltage results in a larger source function for electron-ion pair generation near the cathode, because the ionization mean free path is smaller at these lower beam voltages. Typically, the voltage results in beam energies yielding electron-ion pair source functions near the maximum value in helium. Also, the lower operating voltage tends to lessen the arcing which sometimes occurs near the glass container at the cathode. However, the disadvantage of the lower operating voltage is that the primary beam-reaching distance is smaller than that of the conventional brush cathode discharge, and since electron-ion recombination is the dominant electron loss mechanism, this results in a substantial decay in the secondary electron number density in the axial direction away from the cathode. This effect occurs for all discharge currents up to $900 \mathrm{~mA}$ at pressures above 0.8 torr and at sufficiently low voltage and current for pressures below this value. The beam-reaching distance can be calculated using (ref. 2)

$$
L=\frac{I}{p V_{i}} \int_{0}^{V} \frac{d V}{\beta \phi(V)}
$$

where $\phi(V)$ is the inverse ionization mean free path, $\beta$ is a measure of the ionization efficiency, $p$ is the pressure in torr, $\mathrm{V}_{i}$ is the ionization potential of helium, and $\mathrm{V}$ is the cathode fall voltage which is assumed to be equal to the discharge voltage. Using $\beta=3.0$ and assuming $\phi(V)=C / V$, where $C \sim 430$ in helium, the beam-reaching distances for various currents and pressures are shown in Table $I$. Only for beam-reaching distances less than $15 \mathrm{~cm}$ is a substantial effect of beam decay on the axial variation of secondary electron number density expected. Results

*This is of particular importance for this experiment since the cathode is so large. 
TABLE I

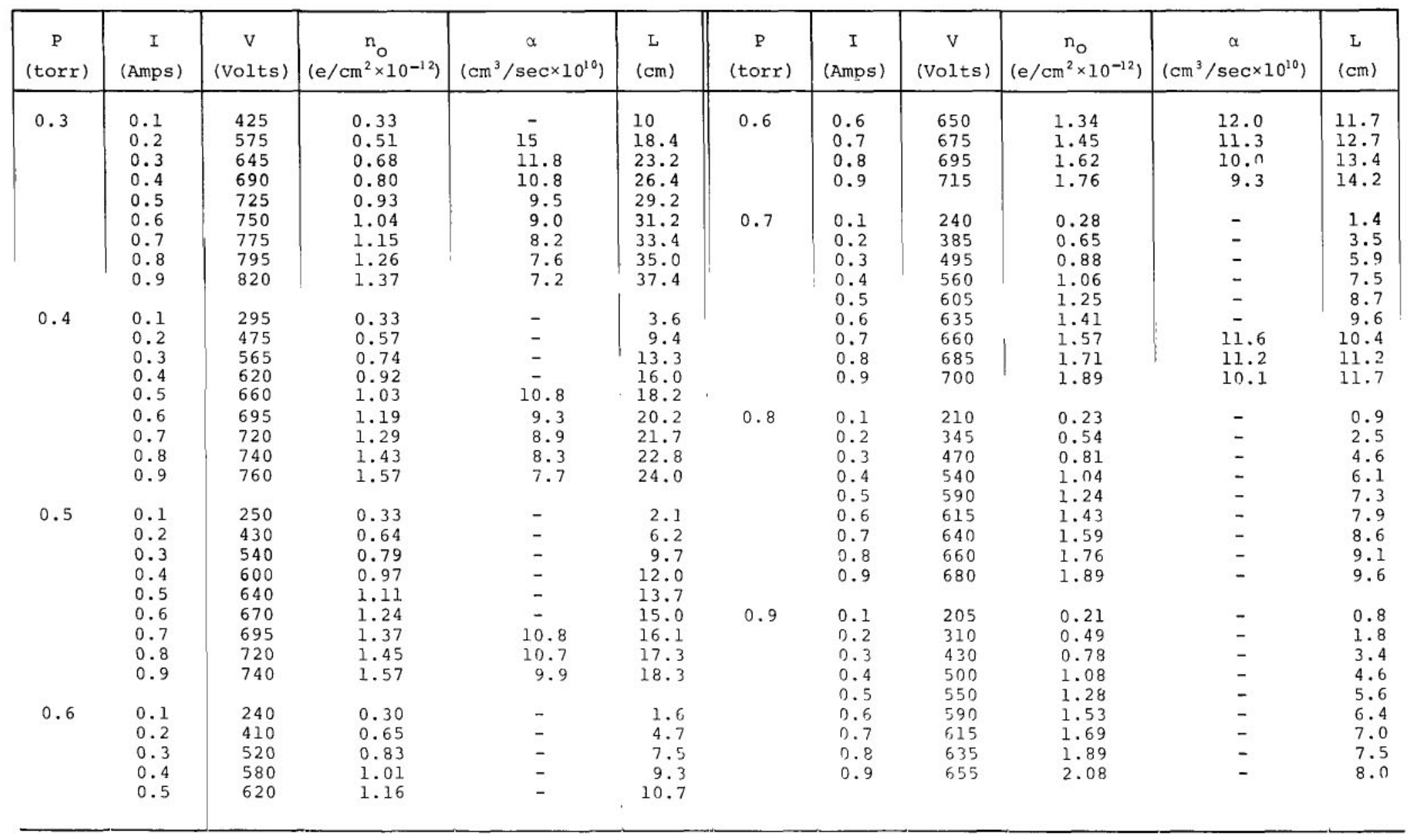


which are consistent with this argument will be presented later. These results assume:

(1) $\beta$ is constant with electron energy;

(2) The ionization mean free path varies inversely with electron energy;

(3) The beam is monoenergetic at the cathode.

Assumption (1) is obviously incorrect but, since $\beta$ is not known accurately at any energy except that it lies between 1 and 10, the use of $\beta=3.0$ for all energies appears to be reasonable for the qualitative argument which is presented. Assumptions (2) and (3) are also questionable at the low beam voltages used in this experiment. Hence, the beam-reaching distances shown in Table I are, at best, crude approximations but they will. be used to support the hypothesis that the V-groove cathode results in a beam-generated plasma.

Finally, the visual appearance of the discharge is described. At applied voltages comparable to the normal cathode fall voltage, a weak blue-green glow appears in the chamber. As the voltage is increased above this value, the glow quickly changes to a deep pink color in the center of the discharge region and is surrounded by a blue-green boundary near the electrodes and the glass wall. The thickness of this boundary decreases with increasing pressure. The pink color is indicative of recombination in helium, and the blue-green boundary defines the region where the primary electron loss mechanism changes from volume recombination to ambipolar diffusion to the walls. For small operating voltages at the lower pressures (i.e., 0.4 to 0.8 torr) and for all operating voltages at higher pressures, the glow is most intense near the cathode. At high operating voltages and currents and at pressures less than 0.8 torr, the discharge is visually symmetrical relative to the center of the chamber.

\section{MEASUREMENTS OF THE PLASMA PARAMETERS}

A movable Langmuir probe was used to obtain electron density and temperature as a function of position, discharge current, and gas pressure. For the range of these parameters over which the discharge was operated, the electron temperature is constant. A typical set of Langmuir probe current-voltage curves at a fixed pressure ( 0.6 torr) and a fixed position (near the center of the discharge chamber) for discharge currents ranging from 0.1 to 0.9 amp is shown in Figure 3. Since the saturation electron current can be determined quite accurately from these data and the electron temperature is constant, the reduction of these curves to semilog 


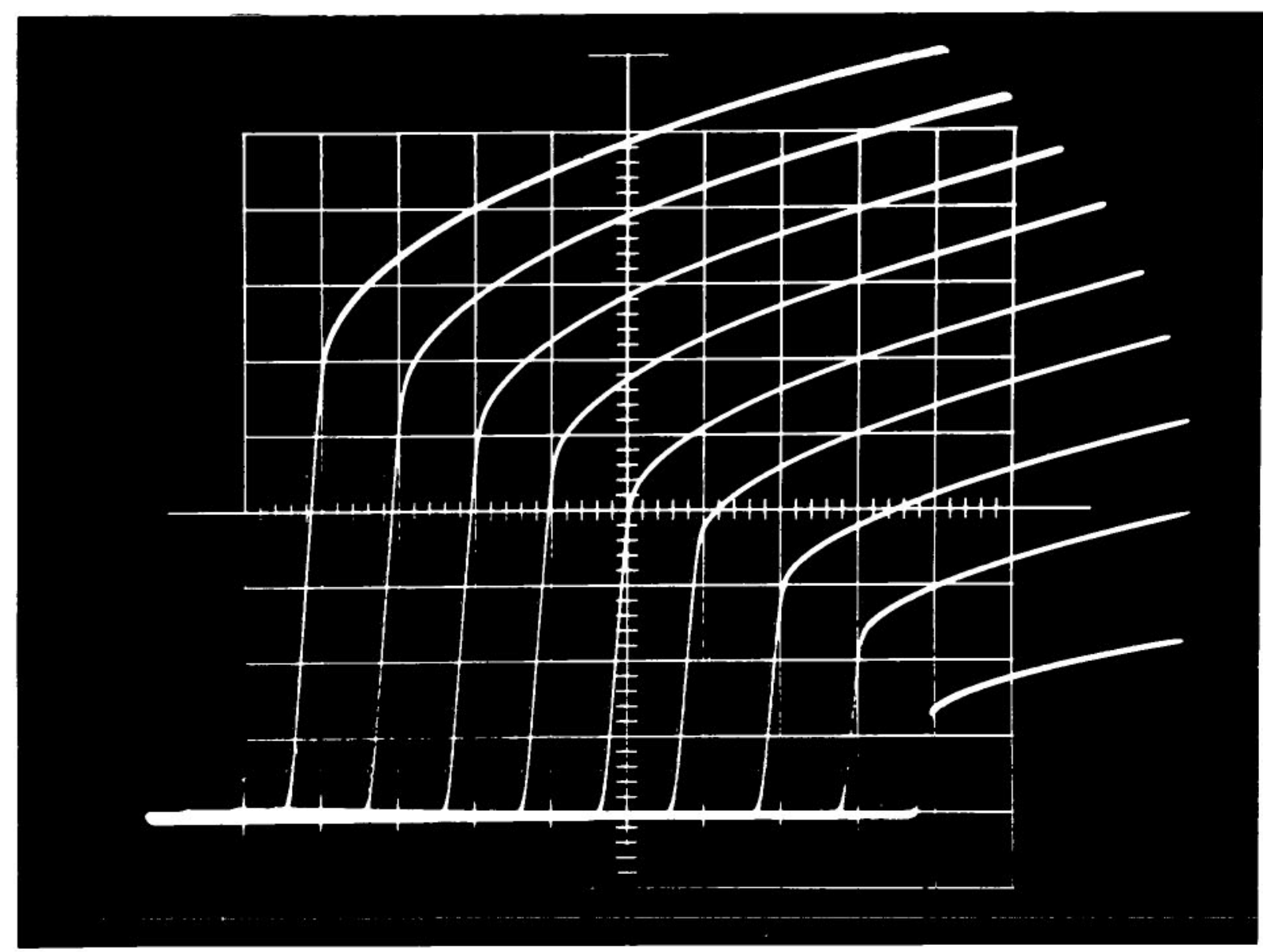

Figure 3.- Typical Langmuir probe current-voltage curves for a probe located at the center of the discharge chamber for $p=0.6$ torr and for discharge currents from 0.1 to $0.9 \mathrm{amp}$

curves was not done in the bulk of the data reduction. Figures 4 through 10 show electron density profiles in the axial direction for various pressures and discharge currents. The independent variable is normalized to 3 inches and the electron density is normalized to its peak value. Figures 11 through 17 show the peak electron density as a function of discharge current for various pressures. The radial electron density profile was also obtained but is not of much concern to the results of this investigation. Previous investigations (refs. 2, 4) have shown that Langmuir probe data are questionable in a beam-generated plasma. No such difficulty was encountered with the V-groove cathode except when the probe was placed quite near the cathode (i.e., within the blue-green boundary near the electrode). It is believed that this is due to the lower operating voltage of the discharge which gives a less energetic beam. 

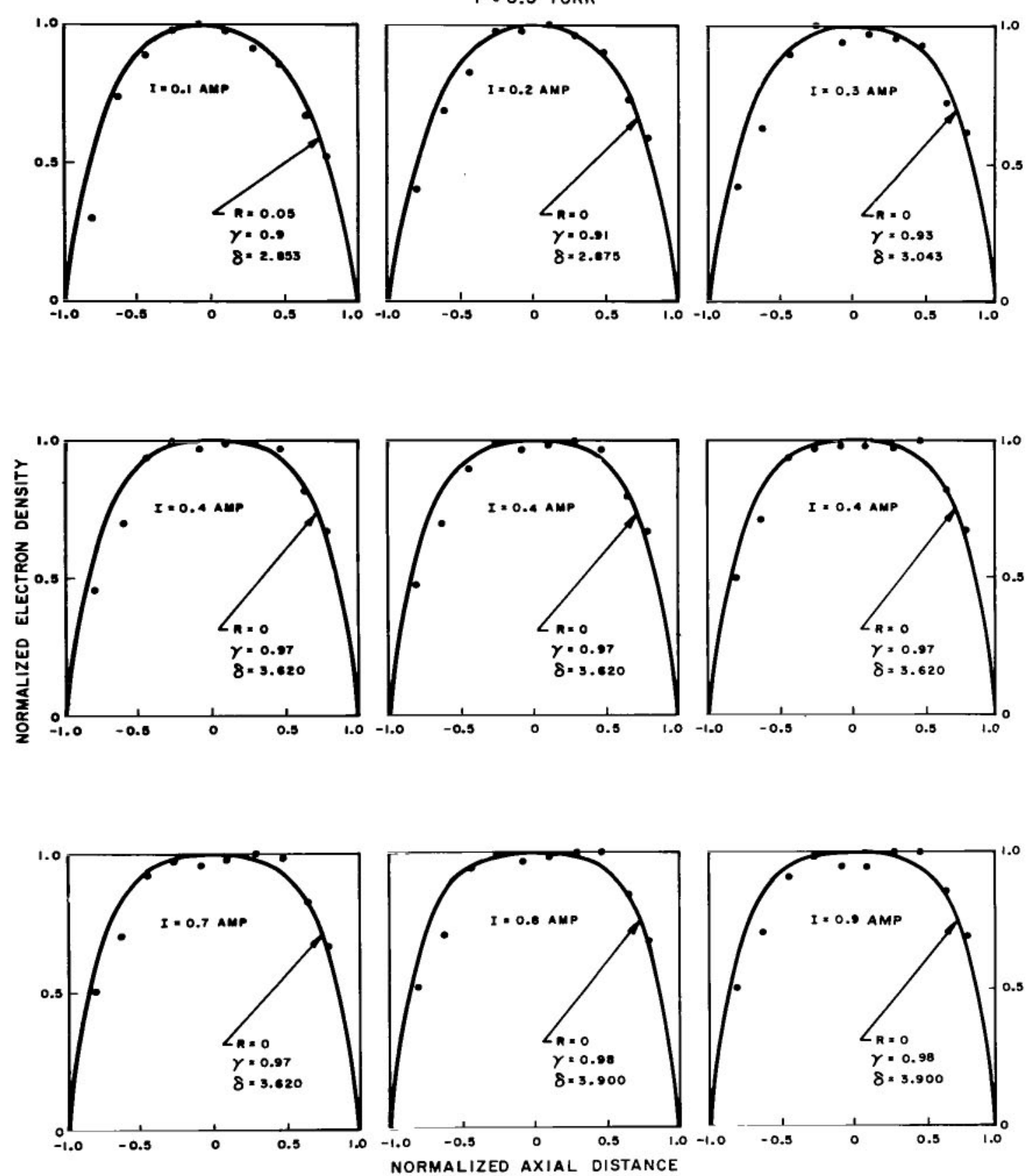

Figure 4.- Electron density profiles for $p=0.3$ torr and discharge currents ranging from 0.1 to 0.9 amp; comparison of theoretical curves and experimental points. 

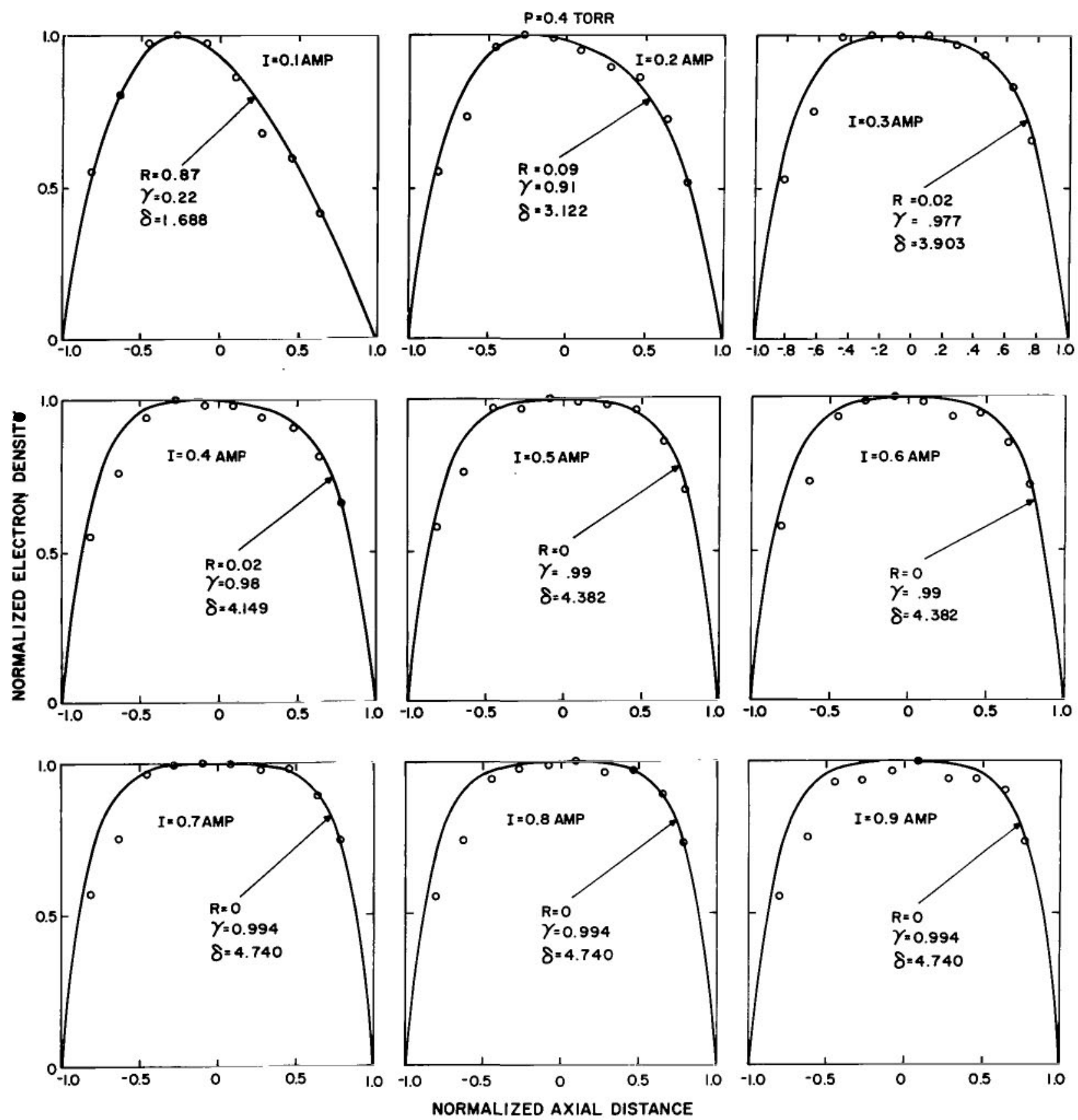

Figure 5.- Electron density profiles for $p=0.4$ torr and discharge currents ranging from 0.1 to 0.9 amp; comparison of theoretical curves and experimental points. 

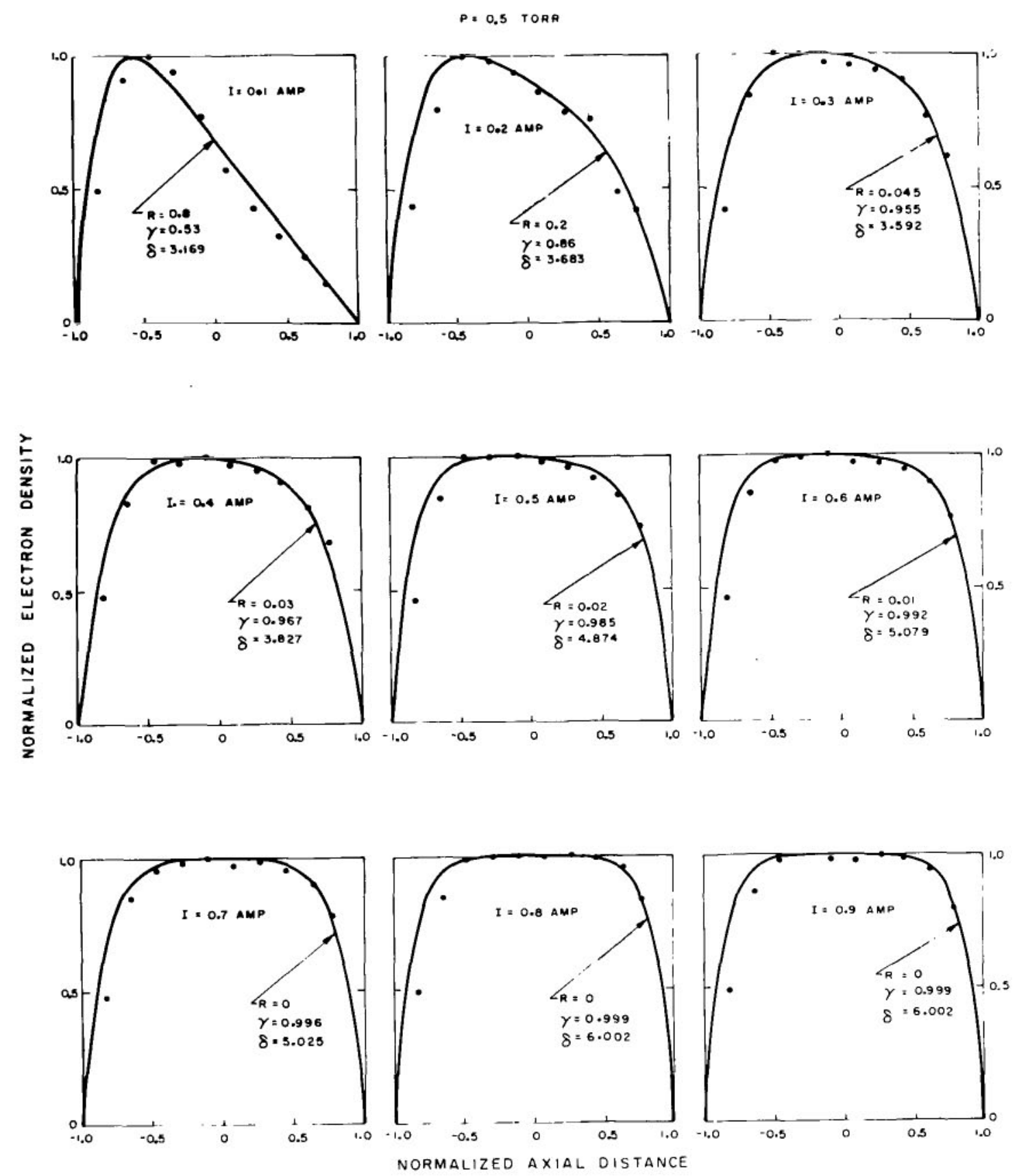

Figure 6.- Electron density profiles for $p=0.5$ torr and discharge currents ranging from 0.1 to 0.9 amp; comparison of theoretical curves and experimental points. 

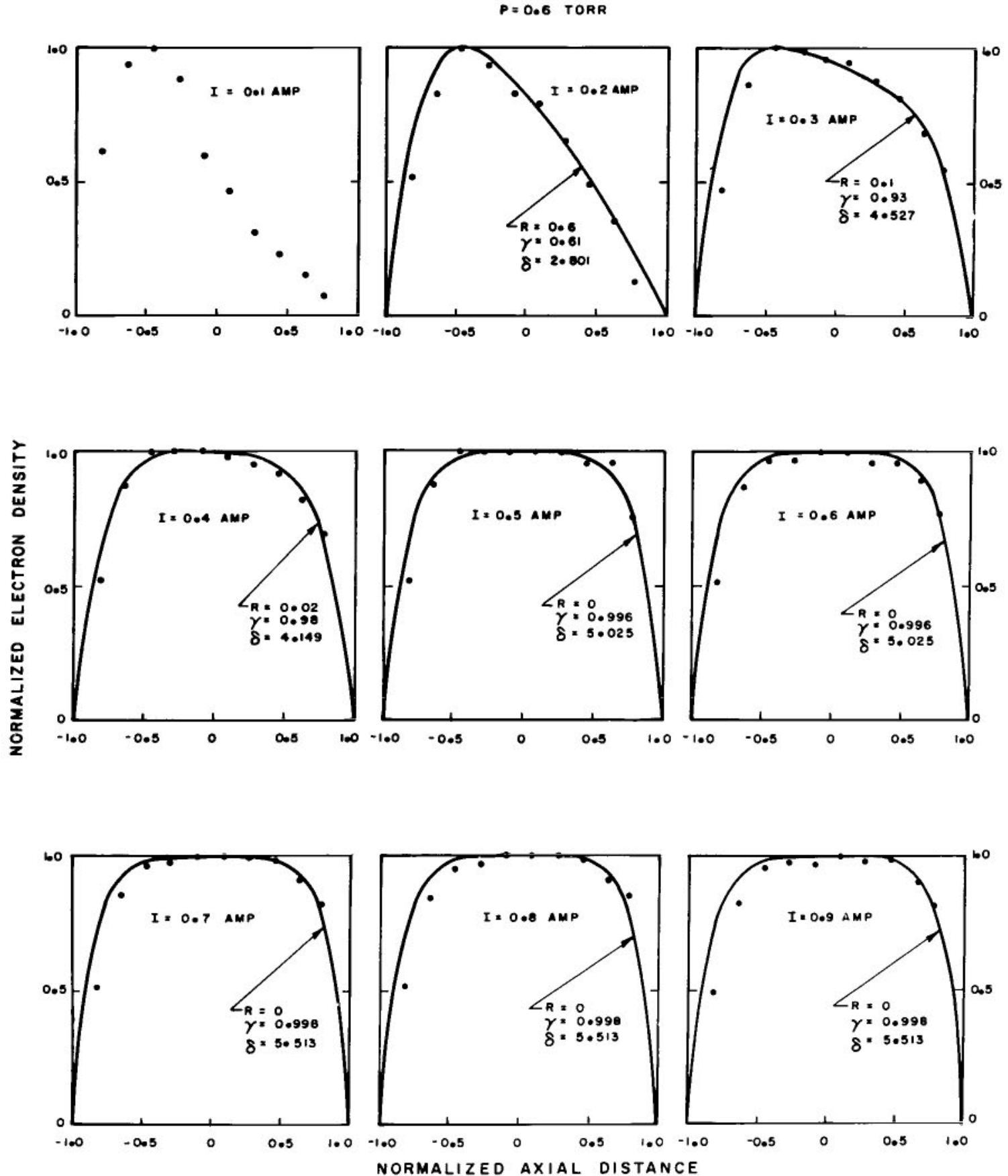

Figure 7.- Electron density profiles for $p=0.6$ torr and discharge currents ranging from 0.1 to 0.9 amp; comparison of theoretical curves and experimental points. 
$=0.7$ TORR
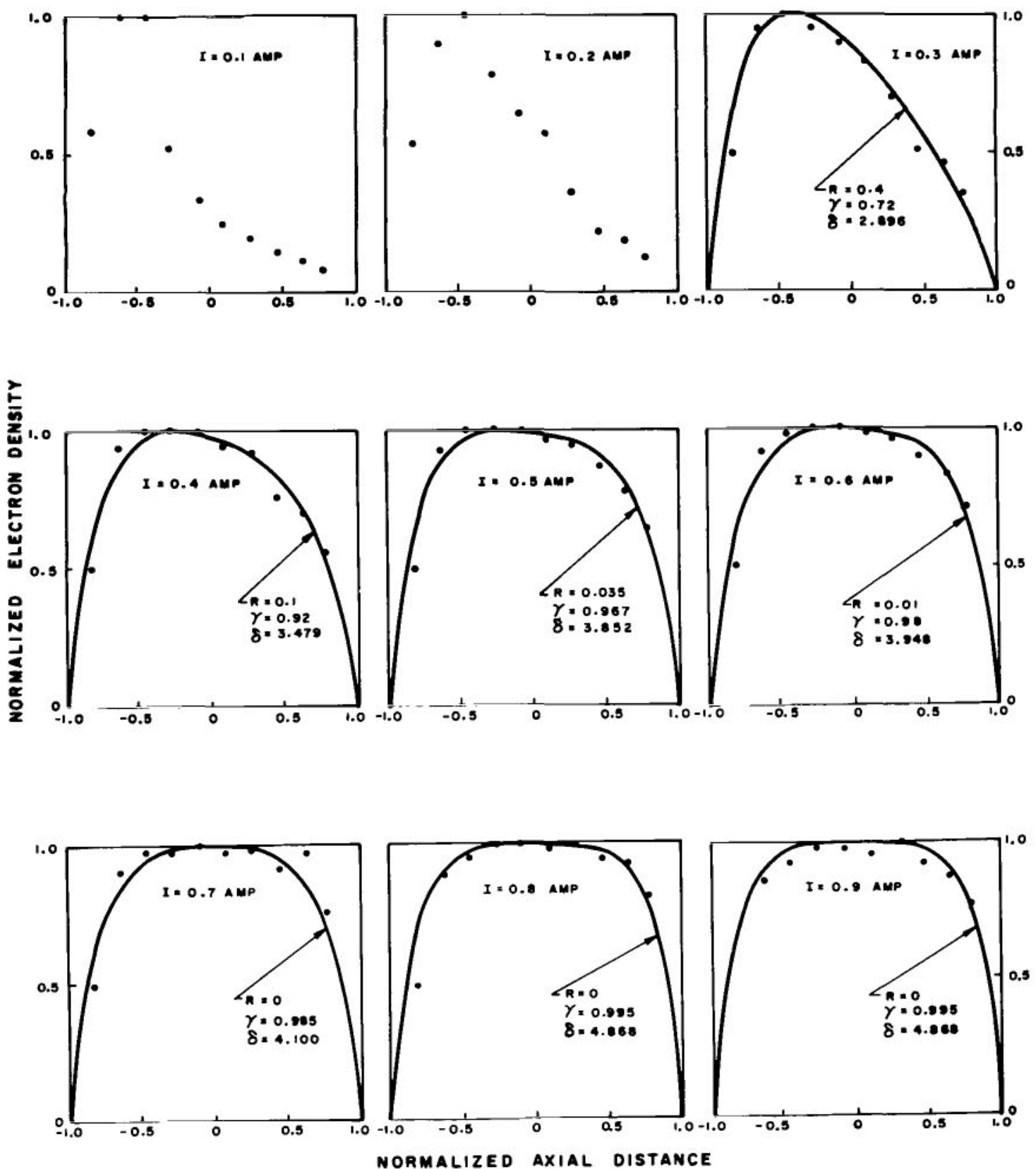

Figure 8.- Electron density profiles for $p=0.7$ torr and discharge currents ranging from 0.1 to 0.9 amp; comparison of theoretical curves and experimental points. 

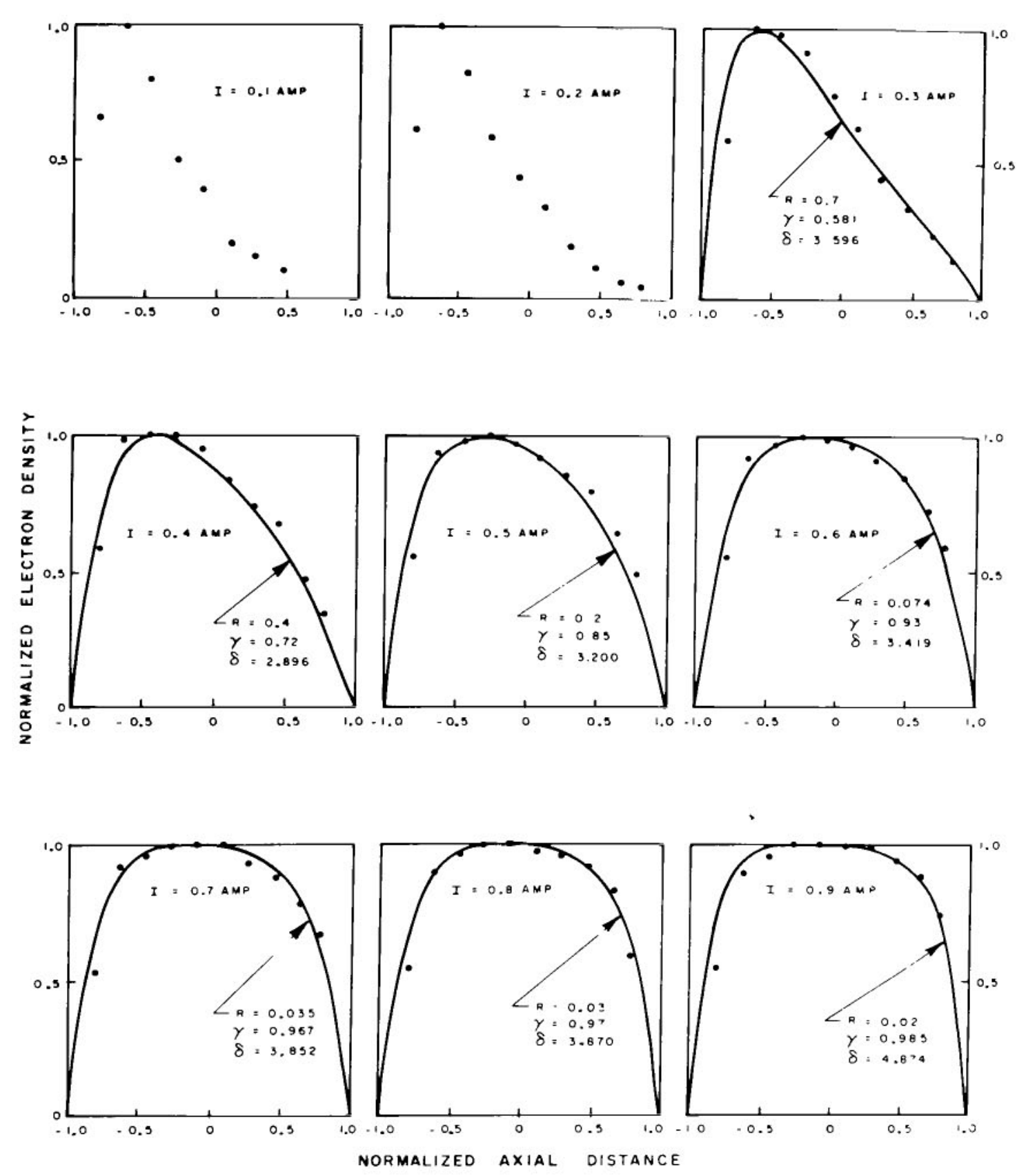

Figure 9.- Electron density profiles for $p=0.8$ torr and discharge currents ranging from 0.1 to 0.9 amp; comparison of theoretical curves and experimental points. 



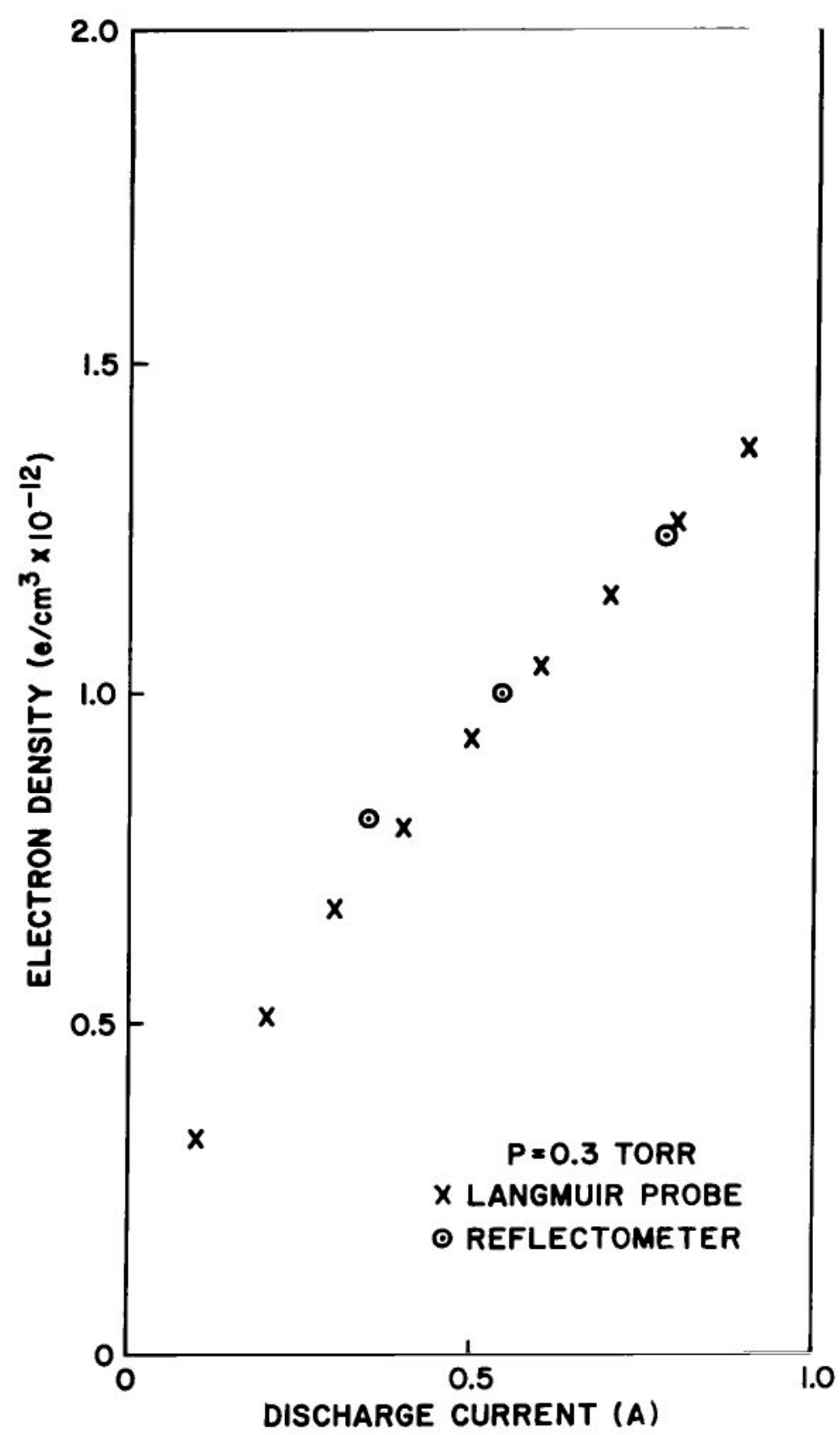

Figure 11.- Comparison of the peak electron densities measured by the Langmuir probe and the electron densities obtained from the reflectometer as a function of discharge current for $\mathrm{p}=0.3$ torr. 


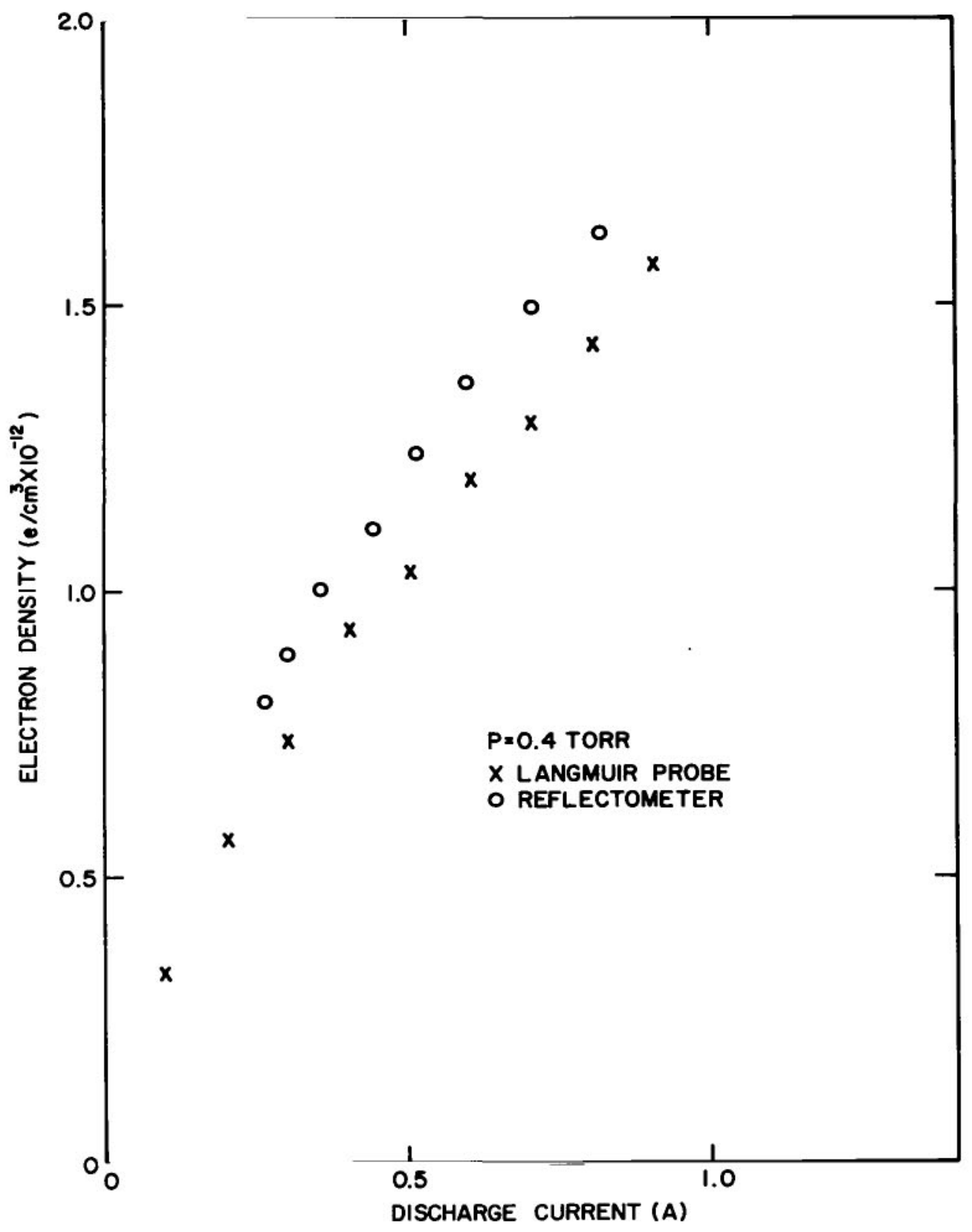

Figure 12.- Comparison of the peak electron densities measured by the Langmuir probe and the electron densities obtained from the reflectometer as a function of discharge current for $\mathrm{p}=0.4$ torr. 


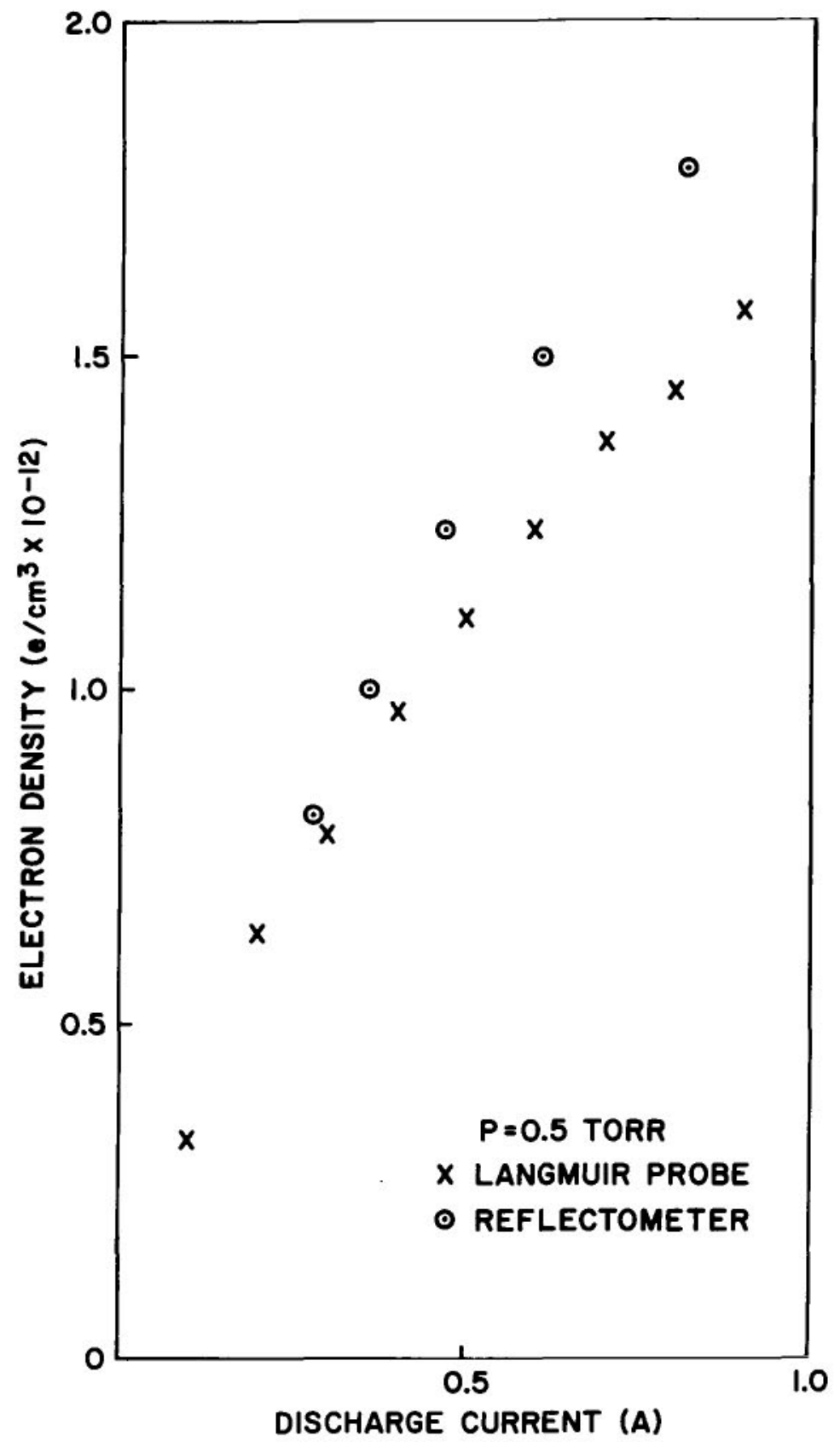

Figure 13.- Comparison of the peak electron densities measured by the Langmuir probe and the electron densities obtained from the reflectometer as a function of discharge current for $\mathrm{p}=0.5$ torr. 


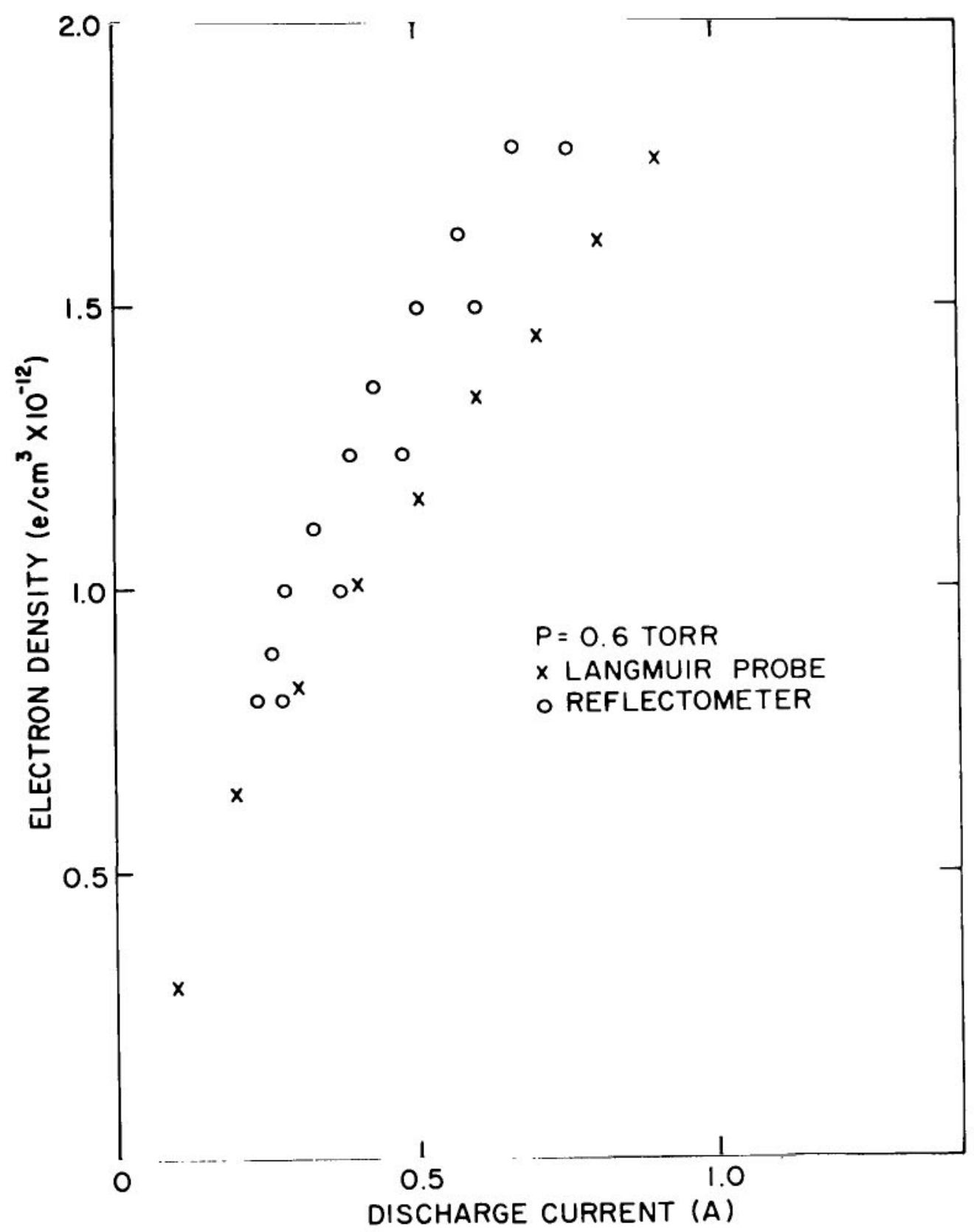

Figure 14.- Comparison of the peak electron densities measured by the Langmuir probe and the electron densities obtained from the reflectometer as a function of discharge current for $\mathrm{p}=0.6$ torr. 


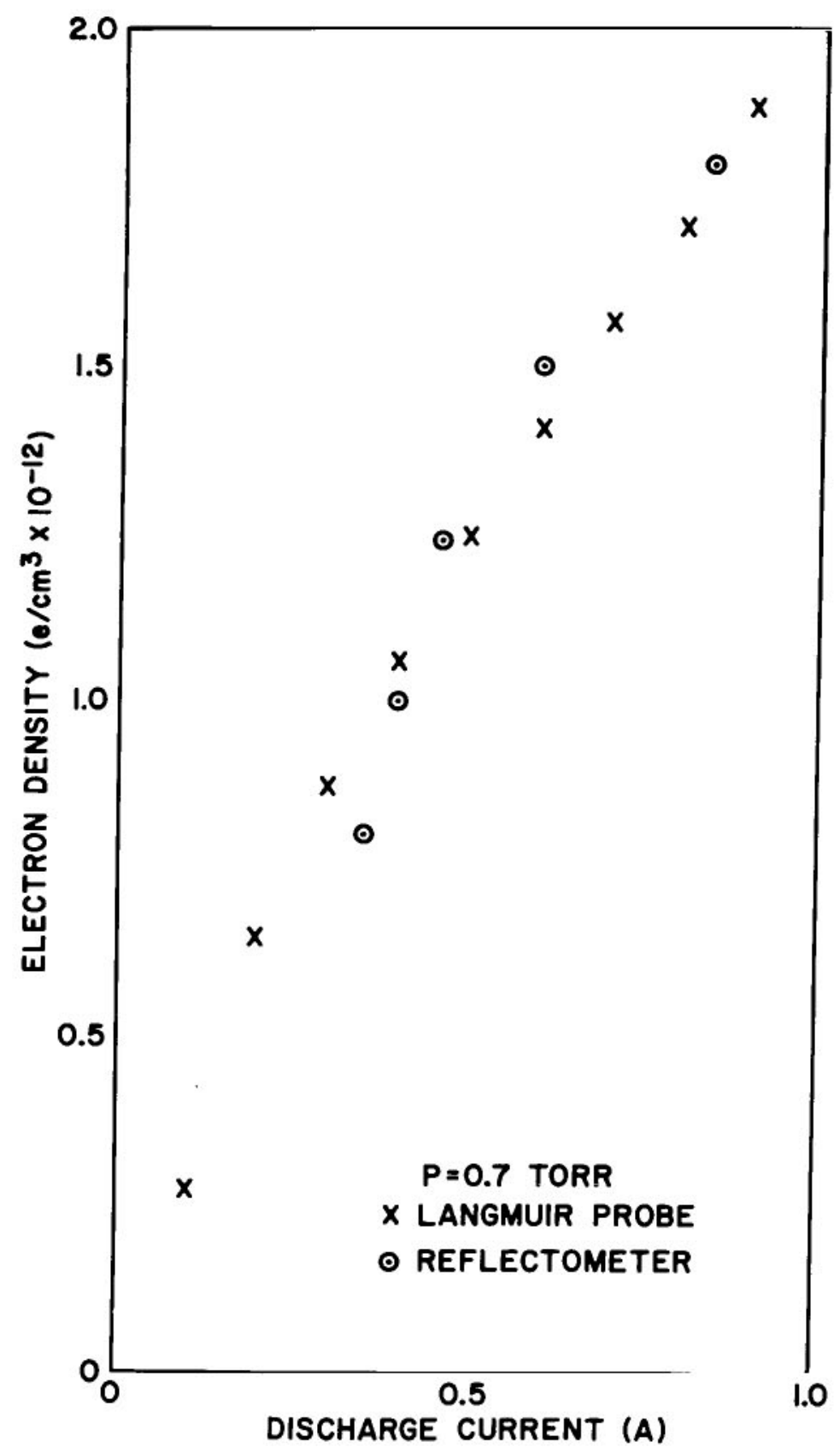

Figure 15.- Comparison of the peak electron densities measured by the Langmuir probe and the electron densities obtained from the reflectometer as a function of discharge current for $\mathrm{p}=0.7$ torr. 


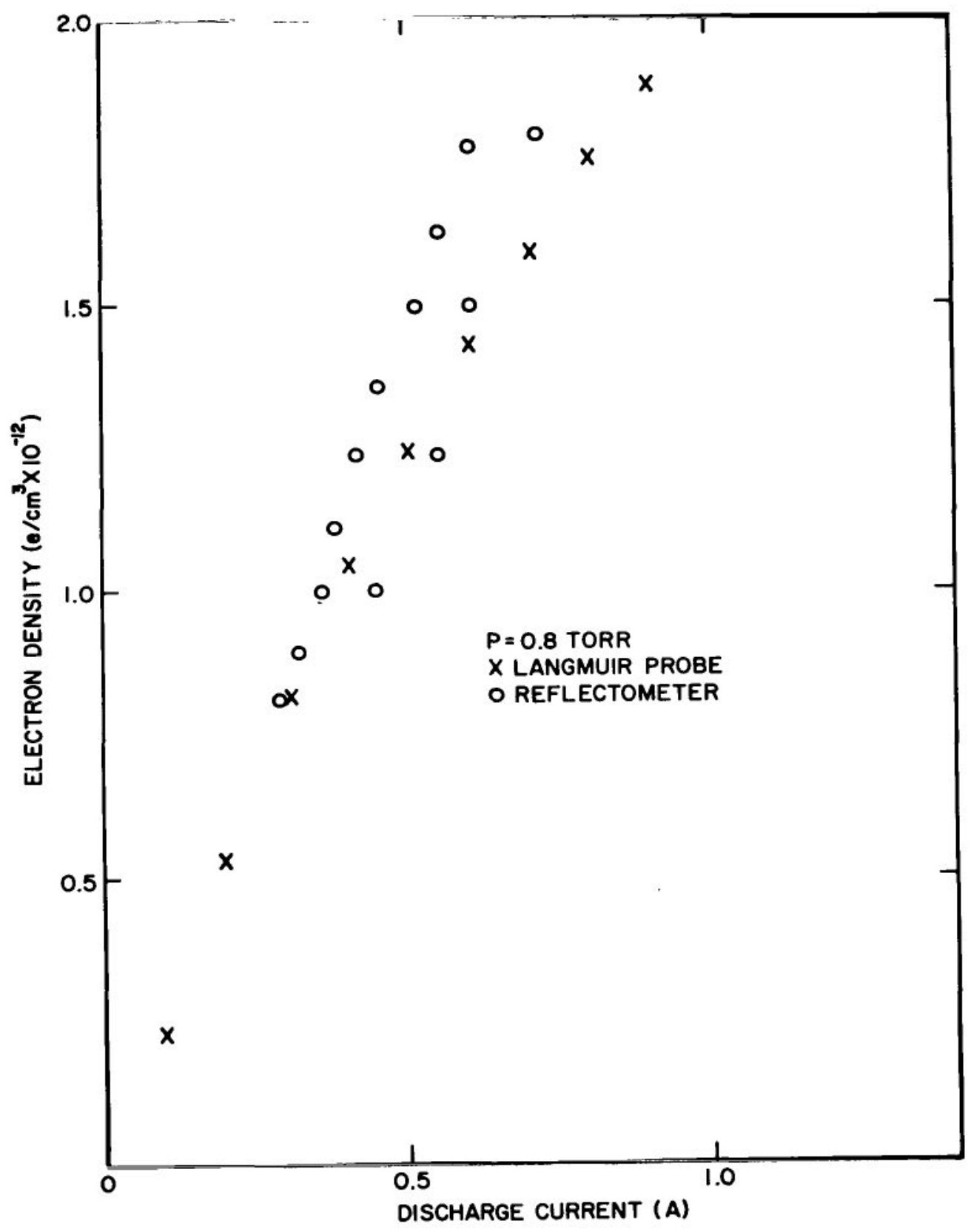

Figure 16.- Comparison of the peak electron densities measured by the Langmuir probe and the electron densities obtained from the reflectometer as a function of discharge current for $\mathrm{p}=0.8$ torr. 


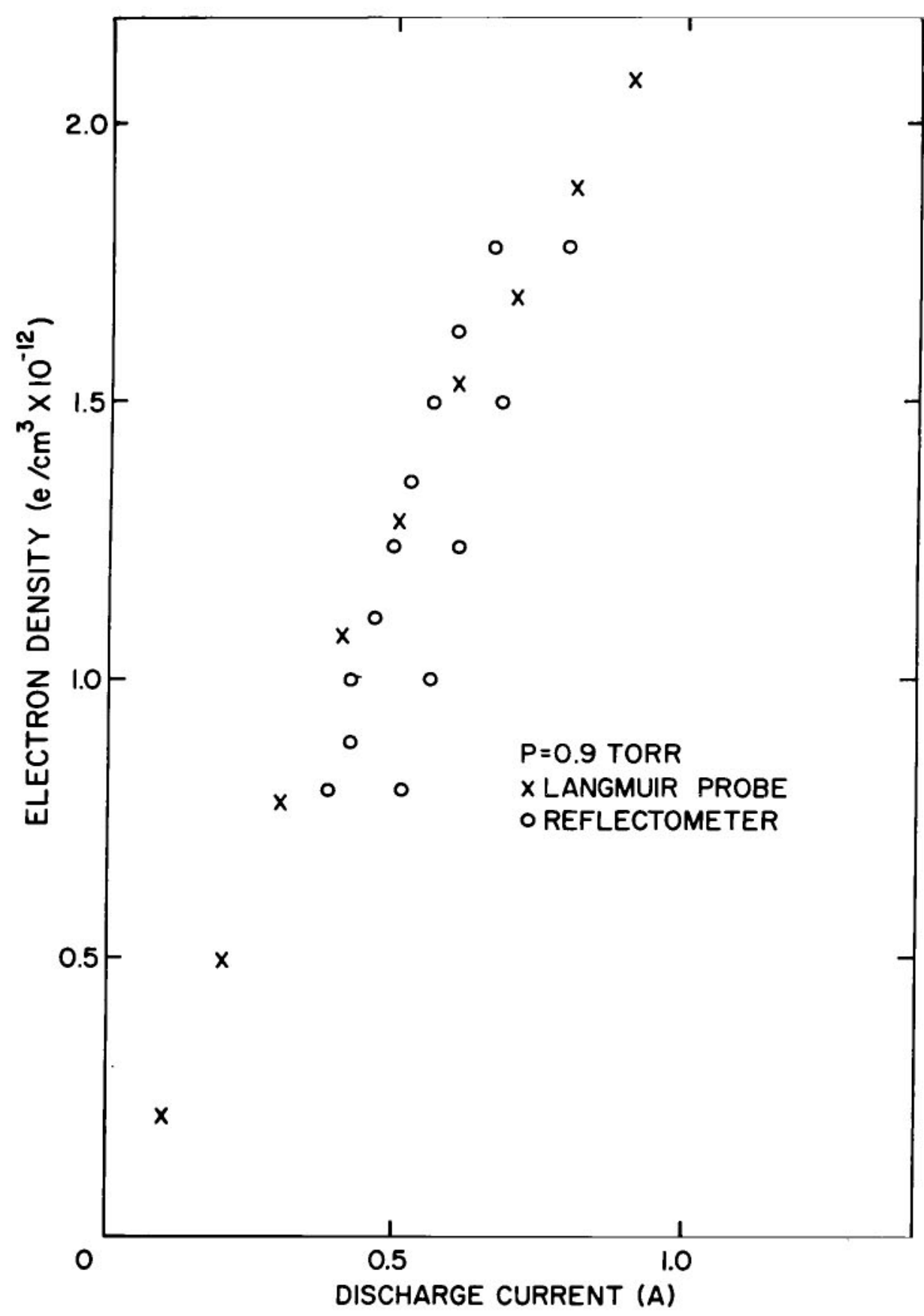

Figure 17.- Comparison of the peak electron densities measured by the Langmuir probe and the electron densities obtained from the reflectometer as a function of discharge current for $\mathrm{p}=0.9$ torr. 
An $\mathrm{X}$-band microwave reflectometer was connected to an openended waveguide port positioned in the center of the anode. The reflection coefficient of the open-ended guide plus plasma layer was monitored as a function of discharge current for fixed frequency and as a function of frequency for fixed discharge current. A typical curve of reflection coefficient as a function of discharge current is shown in Figure 18. In either case, the transition from low to high reflection is well defined and determines quite accurately the condition $\omega_{p}=\omega$, where $\omega_{p}$ is the plasma frequency and $\omega$ is the wave frequency of the reflectometer. Except for operating conditions where there is a sizable beam decay, the plasma frequency is described by the peak electron density. The electron densities obtained by this method are compared with the peak densities measured by the probe in Figures 11 through 17. The agreement is consistent with the accuracies of the two measurement techniques.

There is no accurate method for measuring the collision frequency of a large, dense plasma. However, from knowledge of the electron temperature, the gas type and pressure, and the ion density, it is possible to calculate the collision frequency. The electron-neutral collision frequency is determined from published data on the collision cross-section for momentum transfer in helium (ref. 5) using

$$
\nu_{\text {en }}=100 p P_{c} \sqrt{\frac{2 \mathrm{kT}_{\mathrm{T}}}{\mathrm{m}_{\mathrm{e}}}}
$$

where $\mathrm{p}$ is the pressure in torr, $\mathrm{P}_{\mathrm{C}}$ is the collision probability in inverse $\mathrm{cm}$ at 1 torr, $\mathrm{k}$ is Boltzmann's constant, $\mathrm{T}_{e}$ is the electron temperature, and $\mathrm{m}_{e}$ is the electron mass. For the measured parameters of the discharge, ven ranges from about $1.5 \times 10^{8}$ to $4.5 \times 10^{8}$ for pressures between 0.3 and 0.9 torr. At the relatively low electron temperature and high electron densities generated by the V-groove cathode discharge, electron-ion collisions are expected to be important (refs. 2, 6). To verify this, the following expression for the electron ion collision frequency is used (ref. 5):

$$
\nu_{e i}=\left(\frac{2}{\pi}\right)^{1 / 2} \omega_{p} \frac{\ln \left(9 n_{D}\right)}{9 n_{D}}
$$

where $\omega_{p}$ is the plasma frequency and $n_{D}$ is the number of electrons in a Debye sphere. For electron densities corresponding to $\mathrm{x}$-band cut-off frequencies and the temperature of this experiment, this 


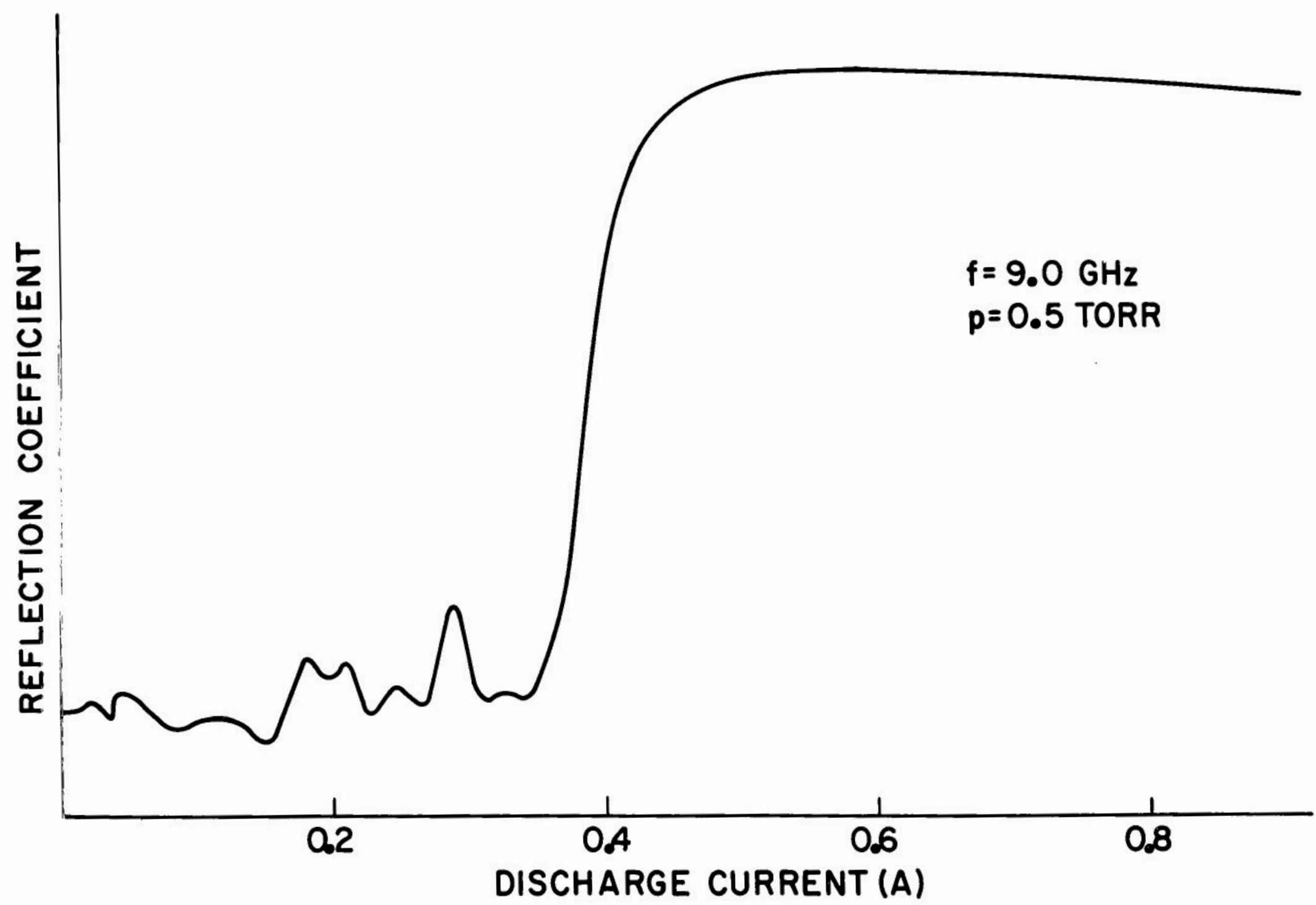

Figure 18.- Typical reflectometer output showing reflection coefficient as a function of discharge current. 
expression gives values larger than the electron neutral collision frequency by a factor of about 2 to 3 depending on the pressure. Of course, it is questionable whether expression (3) is accurate to this factor. In any event, it is expected that $v_{e i}$ is at least comparable to $v_{e n}$. The total collision frequency is

$$
\nu_{t}=\nu_{e n}+\nu_{e i}
$$

\section{THE ONE-DIMENSIONAL DIFFUSION-RECOMBINATION THEORY}

Qualitative arguments have been presented to show that the plasma is beam generated. The electrons in this beam emanate from the cathode and are rapidly accelerated in the cathode fall region. This region is assumed to be very short compared to the anode-cathode separation; that is, the beam electrons leave the cathode with energy corresponding to the full cathode fall voltage which is assumed equal to the discharge voltage. The beam electrons produce the plasma electrons and ions by impact ionization.* The expression $\phi(V)$ is defined as the inverse ionization mean free path at 1 torr for an electron of energy $V$, so that the number of electron-ion pairs generated per second per unit volume, hereafter called the source function for electron-ion pairs, is,

$$
\mathrm{S}=\frac{\mathrm{pJ}}{\mathrm{e}} \phi(\mathrm{V})
$$

where $\mathrm{J}$ is the beam current density which is assumed equal to the discharge current density, $\mathrm{p}$ is the discharge pressure in torr, and $e$ is the electron charge. The beam energy at any axial point, $z$, is the initial beam energy minus the ionization and excitation energies expended in reaching the point in question. A onedimensional planar problem which neglects variations in the radial direction will be considered. This is a good approximation for determining the axial electron density profile, since the cylinder diameter is twice the electrode spacing.

It is difficult to obtain a good functional representation for $S(z)$ in terms of the discharge voltage, current, and position $z$. Instead, consider the source function to be known and some idealized cases are treated later. If electrons and ions

*It can easily be shown that the plasma density is orders of magnitude greater than the beam electron density. 
are lost by recombination and ambipolar diffusion, then, in the steady state:

$$
D \frac{d^{2} n}{d z^{2}}-\alpha n^{2}+s(z)=0, \quad-d \leq z \leq d,
$$

where $D$ is the ambipolar diffusion coefficient, $\alpha$ is the recombination coefficient, and $\mathrm{n}$ represents either the electron or ion number density. Both $\mathrm{D}$ and $\alpha$ are assumed to be independent of position and the following boundary conditions are used:

$$
n(d)=n(-d)=0 \text {. }
$$

Equation (6) may be written:

$$
\frac{d^{2} f}{d x^{2}}+\delta^{2}\left[s(x)-\gamma f^{2}\right]=0
$$

where

$$
\begin{aligned}
& \mathrm{f}=\mathrm{n} / \mathrm{n}_{\mathrm{O}}, \\
& \mathrm{x}=\mathrm{z} / \mathrm{d}, \\
& \mathrm{s}(\mathrm{x})=\mathrm{S}_{\mathrm{O}} \mathrm{s}(\mathrm{x}), \\
& \delta^{2}=\frac{\mathrm{S}_{\mathrm{O}} \mathrm{d}^{2}}{\mathrm{D} \mathrm{n}_{\mathrm{O}}}, \\
& \gamma=\frac{\alpha \mathrm{n}_{\mathrm{O}}^{2}}{\mathrm{~S}_{\mathrm{O}}},
\end{aligned}
$$

and $\mathrm{n}_{\mathrm{O}}$ is the peak electron density and $\mathrm{S}_{\mathrm{O}}=\mathrm{S}(\mathrm{O})$.

First consider the special case $s(x)=1$. This is a good approximation to the experimental situation when the beamreaching distance is large compared to the distance $2 \mathrm{~d}$. Thus, 
the source function is constant and the solution is symmetrical about $\mathrm{x}=0$, where the electron and ion densities are maximum. With the substitution $\mathrm{y}=\delta \mathrm{x}$, the following expression is obtained.

$$
\frac{d^{2} f}{d y^{2}}+1-\gamma f^{2}=0, \quad 0 \leq y \leq \delta,
$$

subject to the boundary conditions

$$
f(0)=1,\left.\quad \frac{d f}{d x}\right|_{x=0}=0, \quad f(\delta)=0 .
$$

Equation (8) has the first integral

$$
\frac{1}{2}\left(\frac{d f}{d y}\right)^{2}+f-1-\frac{\gamma}{3}\left(f^{3}-1\right)=0 .
$$

Using the substitution $g^{2}=1-f$, the solution of Eq. (9) is written as

$$
y=\sqrt{\frac{6}{\gamma}} \int_{0}^{g} \frac{d \zeta}{\sqrt{\frac{3(1-\gamma)}{\gamma}+3 \zeta^{2}-\zeta^{4}}}
$$

which can be expressed in the form of an elliptic function of the first kind (ref. 7) $F(\theta \backslash \beta)$. Thus

$$
y=\frac{\sqrt{\frac{2}{\gamma}}}{\left[1+\frac{4}{3 \gamma}(1-\gamma)\right]^{1 / 4}} F(\theta \backslash \beta)
$$

where

$$
\tan \beta=\frac{\sqrt{\frac{4}{3 \gamma}(1-\gamma)}}{\sqrt{1+\frac{4}{3 \gamma}(1-\gamma)}-1}
$$




$$
\sin ^{2} \theta=\frac{\frac{4}{3} \sqrt{1+\frac{4}{3 \gamma}(1-\gamma)} g^{2}}{\left[1+\sqrt{\frac{4}{3 \gamma}(1-\gamma)}\right]\left[\sqrt{1+\frac{4}{3 \gamma}(1-\gamma)}-1+\frac{2}{3} g^{2}\right]} \cdot
$$

The quantity $\delta$ is obtained from

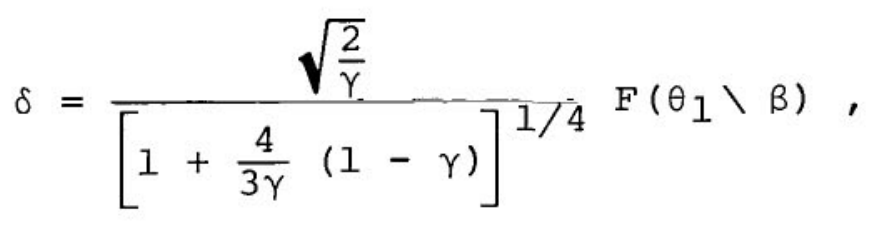

where

$$
\sin ^{2} \theta_{1}=\frac{4 \sqrt{1+\frac{4}{3 \gamma}(1-\gamma)}}{\left[1+\sqrt{\frac{4}{3 \gamma}(1-\gamma)}\right]\left[1+3 \sqrt{1+\frac{4}{3 \gamma}(1-\gamma)}\right]} .
$$

Figure 19 shows normalized electron density as a function of normalized axial distance for various values of $\gamma$. The value $\gamma=0$ is the zero recombination limit. The limit of zero diffusion where all electrons are lost by recombination is $\gamma=1$, and $\delta=\infty$, where $\mathrm{f}=1$, for $0 \leq \mathrm{x}<1$. From Figure 19, it is noted that even when $\gamma$ is quite near 1 so that nearly all the electron loss is by recombination, the profile is still greatly influenced by diffusion.

When the electron-ion pair source function is not constant, the functional behavior of $\mathrm{S}(\mathrm{z})$ must be known to obtain solutions of Eq. (6). However, there is probably no one functional form of $\mathrm{S}(\mathrm{z})$ which is valid for all discharge voltages and currents. In order to display in a qualitative fashion that beam attenuation can account for the asymmetrical profiles which occur at sufficiently low voltage or sufficiently high pressure, the secondorder, non-linear differential Eq. (6) will be solved with

$$
S(z)=S_{O} e^{-z / L}
$$




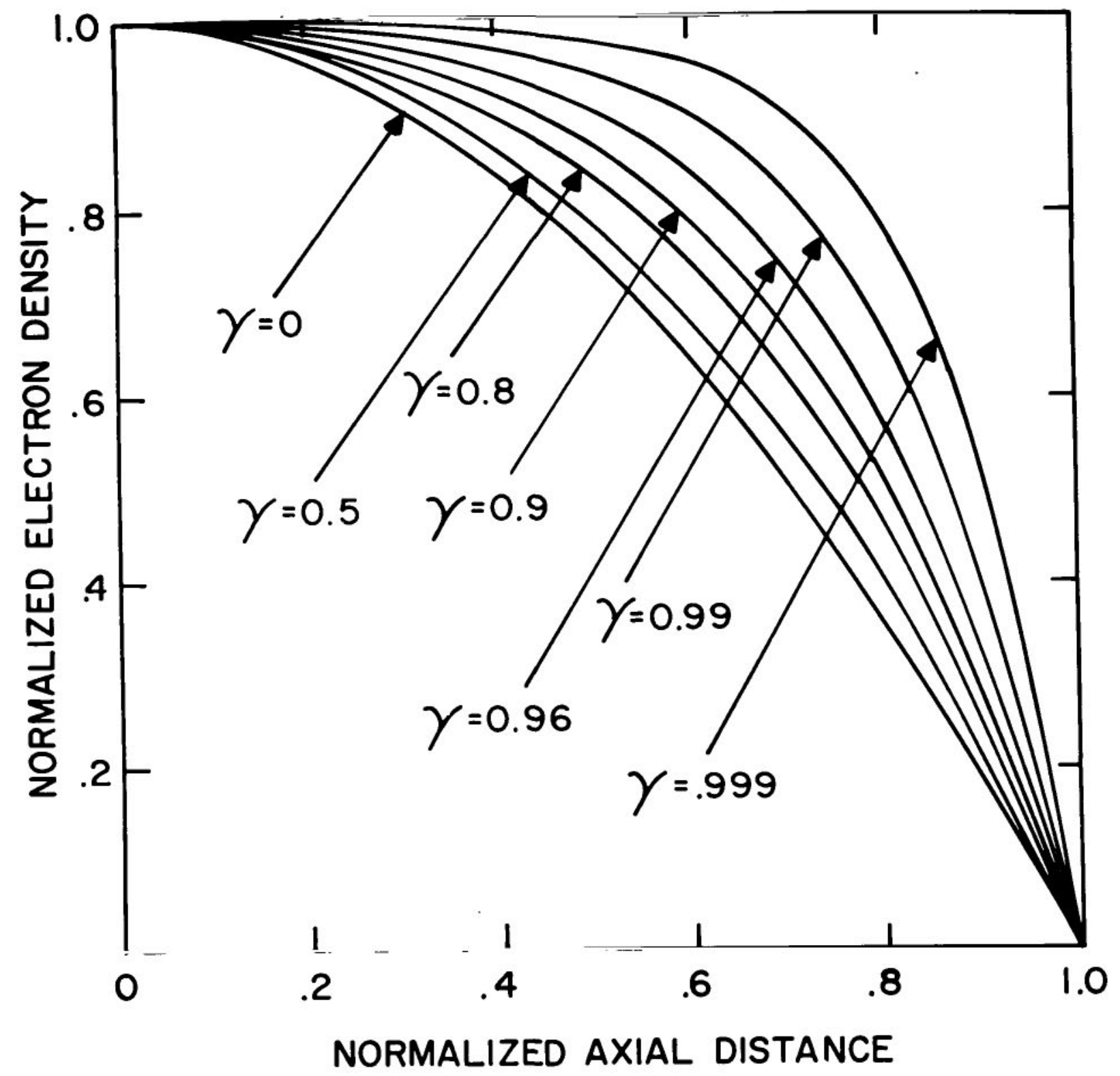

Figure 19.- Theoretical electron density profiles showing normalized electron density as a function of normalized axial distance for various values of $\gamma$. 
Hence Eq. (7) becomes

$$
\frac{d^{2} f}{d x^{2}}+\delta^{2}\left[e^{-(d / L) x}-\gamma f^{2}\right]=0,
$$

and solutions of this equation must be obtained numerically. To facilitate the numerical procedure, substitute

$$
y=\delta_{1}\left(x+x_{1}\right)
$$

where $x_{1}$ is defined such that

$$
\left.\frac{d n}{d x}\right|_{x=-x_{1}}=0, \quad n\left(-x_{1}\right)=n_{0}
$$

Hence

$$
\frac{d^{2} f}{d z^{2}}+e^{-R y}-\gamma_{1} f^{2}=0,
$$

where

$$
\begin{aligned}
& \gamma_{1}=\gamma e^{-(d / L) x_{1},} \\
& \delta_{1}^{2}=\delta^{2} e^{(d / L) x_{1},} \\
& R=\frac{d}{\delta_{1} L},
\end{aligned}
$$

and the boundary conditions are

$$
f\left[\delta_{1}\left(1+x_{1}\right)\right]=f\left[\delta_{1}\left(x_{1}-1\right)\right]=0 .
$$


For given $\mathrm{R}$ and $\gamma_{1}$, Eq. (18) can be integrated numerically for both positive and negative $y$ using the initial conditions $f(0)=1, f^{\prime}(0)=0$, and the boundary conditions yield the constants $\delta_{1}$ and $x_{1}$. These constants are then used to yield solutions in terms of the normalized variable $\mathrm{x}$.

The procedure outlined above for treating the case of a non-constant source function simplifies the numerical computations for finite diffusion. However, the attenuation constant $R$, which is specified in this numerical procedure, only gives the actual constant $L$, once $\delta_{1}$ is determined (i.e., $L=d / R \delta_{1}$ ). Also, the recombination limit is not obtained for finite $R$. That is, the recombination limit for finite $L$ corresponds to

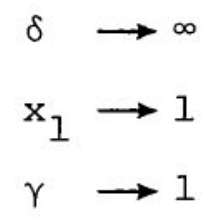

and hence

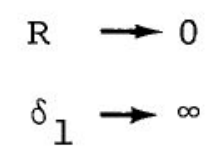

such that $R \delta_{1}$ is finite. In this limit, $\gamma_{1} \rightarrow \exp \left(-R \delta_{1}\right)<1$. However, the numerical procedure is greatly simplified using fixed $R$ and experimentally the full recombination limit is never attained. A best-fit procedure was employed to compare experiment and theory using a sizable number of theoretical curves for wide ranges of $\gamma_{1}$ and $R$, including $R=0$. These compari.sons are shown in Figures 4 through 10. The notations $\gamma$ and $\delta$ are used in these figures for $\gamma_{1}$ and $\delta_{1}$. In view of the experimental error of the Langmuir probe, the inadequacy of the exponential form for approximating the source function and the finite amount of computer data used for matching, these comparisons are made only to support the basic contentions that the plasma is beam-generated and that recombination plays a dominant role in determining the electron densities. Values of L computed from the parameters $R, \gamma$, and $\delta$ for best matching are comparable to the beam-reaching distances computed in the second section (except, of course, for cases where $R=0$ is assumed), but there is no exact correspondence in magnitude, or even in relative values, with changes in either pressure or discharge voltage. 
Finally, the recombination coefficient will be estimated from the measured discharge parameters. For cases where $\mathrm{R} \approx 0$, the values of $\gamma$ which give the better match between theory and experiment are all close to 1. Thus, for a small volume near the center of the discharge, virtually all electron loss is by recombination and

$$
\mathrm{n}_{0} \approx \sqrt{\frac{\mathrm{pJS}(\mathrm{V})}{\mathrm{e} \alpha}},
$$

or

$$
\alpha=\frac{\mathrm{JpS}(\mathrm{V})}{\mathrm{en}_{\mathrm{o}}^{2}} \text {. }
$$

Assuming $\mathrm{V}$ to be the discharge voltage and $\mathrm{J}$ to be the discharge current density, $\alpha$ is calculated. Values of $\alpha$ obtained in this manner are shown in Table I. These values are consistent with the collisional-radiative recombination theory (ref. 3). This theory uses the hydrogen atom, but results for helium should not be significantly different. In any event, it was concluded that the discharge is predominantly recombination-controlled for operating conditions such that $R \approx 0$. No inference should be drawn from the fact that $\alpha$ appears to increase with either decreasing discharge voltage or increasing pressure. This tendency can more likely be attributed to the assumptions concerning the equality of beam and discharge parameters (i.e., voltage and current). As the beam voltage is decreased or the pressure is increased, the contribution of ion flow to the total discharge current is expected to be increasingly important. Also, under these conditions the beam has an increasing spread of electron energies.

\section{CONCLUSIONS}

The results of an experimental investigation of the plasma parameters generated by a large V-groove cathode have been presented. The plasma can be described by the following parameters

$$
\begin{array}{ll}
\mathrm{n}_{\mathrm{e}} \approx 2 \times 10^{12} \mathrm{~cm}^{-3} & \mathrm{~d} \approx 15 \mathrm{~cm} \\
\nu \sim 10^{8}-10^{9} \mathrm{sec}^{-1} & \mathrm{~T}_{\mathrm{e}} \approx 1200^{\circ} \mathrm{K} .
\end{array}
$$


Furthermore, the plasma density is recombination-controlled, and electron-ion collisions play an important role in determining the total collision frequency for momentum transfer.

The results described in this technical note have been confined to discharge currents less than $1.0 \mathrm{~A}$ and an electrode spacing of 6 inches. The discharge has recently been operated with currents up to $5 \mathrm{~A}$ and an electrode spacing of 12 inches. Complete diagnostics are not yet available, but microwave interferometer measurements and reflectometer measurements indicate electron densities greater than $4 \times 10^{12} \mathrm{e} / \mathrm{cm}^{3}$.

Electronics Research Center

National Aeronautics and Space Administration

Cambridge, Massachusetts, March 1969

$125-21-02-80$ 
1. Kaminsky, M.: Atomic and Ionic Impact Phenomena on Metal Surfaces. Academic Press, 1965, pp. 328-329.

2. Persson, K. B.: The Brush Cathode Plasma - A Well Behaved Plasma. J. Appl. Phys., vol. 36, October 1965, pp. 30863094 .

3. Bates, D. R., and Delgarno, A.: Electronic Recombination. Atomic and Molecular Processes (ed. by D. R. Bates), Academic Press, New York, 1962, pp. 245-271.

4. Francis, G.: The Glow Discharge at Low Pressure. Handbuch Der Physik, ed. by S. Flugge, vol. XXII, Springer-Verlag, 1947 , p. 91

5. Shkarofsky, I. P., Johnston, T. W., and Bachynski, M. P.: The Particle Kinetics of Plasmas. Addison-Wesley, 1966.

6. Bekefi, G.: Radiation Processes in Plasmas. Wiley and Sons, 1966.

7. Abramuwitz, M., and Stegun, I. A.: Handbook of Mathematical Functions. NBS Appl. Math. Series, vol. 55, 1964, pp. 587626 . 


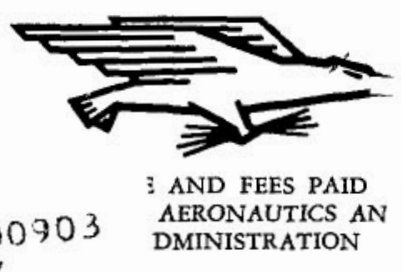

O2U OOL 50 $5130 S$ WEAPONS LABORATORY/AFWLI
AIR FURCE WEAPE
KIRTLAVE AIR FORCE BASE, VEW MEXICO 8711

Ar E. LOU BOWMAN, ACTING CHIEF TECH. LI

POSTMASTER. If Undeliverable (Section 158

$\begin{array}{ll}\text { POSTMASTER: } & \begin{array}{l}\text { If Undeliverable (Section } 158 \\ \text { Postal Manual) Do Not Return }\end{array}\end{array}$

\begin{abstract}
' "The aeronautical and space activities of the United States shall be conducted so as to contribute. . . to the expansion of buman knowledge of phenomena in the atmosphere and space. The Administration shall provide for the widest practicable and appropriate dissemination of information concerning its activities and the results thereof."
\end{abstract}

- National Aeronautics And Space ACt of 1958

\title{
NASA SCIENTIFIC AND TECHNICAL PUBLICATIONS
}

TECHNICAL REPORTS: Scientific and technical information considered important, complete, and a lasting contribution to existing knowledge.

TECHNICAL NOTES: Information less broad in scope but nevertheless of importance as a contribution to existing knowledge.

TECHNICAL MEMORANDUMS:

Information receiving limited distribution because of preliminary data, security classification, or other reasons.

CONTRACTOR REPORTS: Scientific and technical information generated under a NASA contract or grant and considered an important contribution to existing knowledge.
TECHNICAL TRANSLATIONS: Information published in a foreign language considered to merit NASA distribution in English.

SPECIAL PUBLICATIONS: Information derived from or of value to NASA activities. Publications include conference proceedings, monographs, data compilations, handbooks, sourcebooks, and special bibliographies.

\section{TECHNOLOGY UTILIZATION}

PUBLICATIONS: Information on technology used by NASA that may be of particular interest in commercial and other non-aerospace applications. Publications include Tech Briefs, Technology Utilization Reports and Notes, and Technology Surveys.

Details on the availability of these publications may be obtained from:

\section{SCIENTIFIC AND TECHNICAL INFORMATION DIVISION}

NATIONAL AERONAUTICS AND SPACE ADMINISTRATION

Washington, D.C. 20546 